\title{
Immunohistochemical analysis of the mTOR pathway in intrahepatic cholangiocarcinoma
}

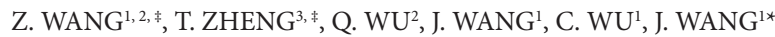 \\ ${ }^{1}$ Department of Oncology, Changzheng Hospital, Second Military Medical University, Shanghai 200433, P.R. China; ${ }^{2}$ Department of Medi- \\ cal Oncology, First Affiliated Hospital, Bengbu Medical College, Anhui 23300, P.R. China; ${ }^{3}$ Cancer Center, Qilu Hospital, Shandong University, \\ Jinan, 250012, P.R. China \\ *Correspondence: jiejunw2@163.com, jiejunw@csco.org.cn \\ ${ }^{*}$ Contributed equally to this work.
}

\section{Received August 24, 2011 / Accepted September 21, 2011}

\begin{abstract}
The aim of the study was to evaluate the expression of activated mammalian rapamycin (mTOR) and its downstream effectors, phosphorylated p70 ribosomal protein S6 kinase (p70S6K) and eukaryotic initiation factor 4E-binding protein 1 (4EBP1), in intrahepatic cholangiocarcinomas (ICC), in order to strengthen the rationale for targeted therapy using mTOR inhibitors in patients with ICC. p-mTOR (Ser 2448), p-4EBP1 (Thr 70) and p-p70S6K (Thr 389) were detected in 77 primary ICC tumors by immunohistochemistry. High levels of p-mTOR, p-4EBP1 and p-p70S6K expression were defined in $48.1 \%$ (37/77), 50.6\% (39/77) and 51.9\% (40/77) of all tumors, respectively. No significant correlation was observed between mTOR pathway proteins overexpression with clinicopathological characteristics and patient's prognosis, except that high p-p70S6K expression correlated with the poorly differentiated subtype, and high expression of $\mathrm{p}-4 \mathrm{EBP} 1$ predicted poor prognosis in ICC patients and retained an independent prognostic factor in multivariate analysis. In conclusion, our results showed high prevalence of activation of mTOR pathway in ICC tumors, suggesting that a high proportion of ICC patients might benefit from mTOR pathway targeted therapies. In addition, p-4EBP1 phosphorylation at Thr 70 could be a useful prognostic biomarker for ICC patients.
\end{abstract}

Key words: intrahepatic cholangiocarcinoma, mTOR pathway, phosphorylation, targeted therapy, prognosis

Intrahepatic cholangiocarcinoma (ICC) is a less frequent disease, and ranks second in malignant liver tumors [1]. In the majority of ICC patients, a definitive diagnosis is established at their advanced stages; hence, the prognosis of these patients is still poor despite of extensive resection of carcinomas [2]. The lack of effective optional therapeutic approaches emphasizes the necessity of identifying novel prognostic factors and therapeutic targets for ICC patients.

The mammalian target of rapamycin (mTOR) pathway, which plays a key role in the cellular growth and homeostasis, is frequently altered in various cancers ${ }^{[3]}$. It has been proved that $\mathrm{mTOR}$ can promote protein synthesis and cell proliferation by phosphorylating p70 ribosomal protein S6 kinase (p70S6K) and eukaryotic initiation factor 4E-binding protein 1 (4EBP1) [4]. Currently, mTOR pathway members have been evaluated as potential therapeutic anticancer targets in several types of cancer [5,6]. Although some clinical trials using mTOR pathway small molecule inhibitors alone or in combination with other agents are on going, there are few evidences that these molecular-targeted treatments are effective in patients with ICC. The aim of the study was to evaluate the expression status and clinicopahological significance of activated mTOR and its downstream effectors in ICC tumors, in order to strengthen the rationale for targeted therapies using mTOR inhibitors in patients with ICC.

\section{Materials and methods}

Patients. Paraffin-embedded tissues from 77 patients with ICC who underwent resection of their tumors at the Changzheng Hospital, Second Military Medical University (Shanghai, China) and First Affiliated Hospital, Bengbu Medical College (Anhui, China), and Qilu hospital Shandong University (Shandong, China) from 1999 to 2006. Having com- 
plete clinicopathological and followed-up data were criteria for patients inclusion in the study. None of the patients had received radio/chemotherapy prior to resection. Patients who had distant metastasis at the time of diagnosis were excluded. Overall survival time (OS) was defined as the time from surgical resection to cancer-related death only. Prior to the start, the study was evaluated and approved by the research ethics committees of our institutions, and the patients' informed consents were also obtained. The clinicopathological details were shown in Table 1.

Immumohistochemistry. $4 \mu \mathrm{m}$ thick of formalin-fixed paraffin-embedded sections were deparaffinized in xylene and rehydrated. After endogenous peroxidase activity blocking (3\% hydrogen peroxide, $20 \mathrm{~min}$ ) and antigen retrieval (boiling in $10 \mathrm{mM}$ citrate buffer, $\mathrm{pH} 6.0$ ), sections were incubated with the primary antibodies overnight at $4^{\circ} \mathrm{C}$. The following primary antibodies were used: rabbit $\mathrm{mAb}$ against $\mathrm{p}-\mathrm{mTOR}$ (Ser 2448) (Cell signaling, 1:100 dilutions), rabbit $m A b$ against $\mathrm{p}-4 \mathrm{EBP} 1$ (Thr 70) (Epitomics, 1:100 dilutions) and mouse $\mathrm{mAb}$ against phospho-p70S6K (Thr 389) (Cell signaling, 1:100 dilutions). Detection was performed with the DakoCytomation Envision Plus peroxidase system using diaminobenzidine (DAB) chromogen as a substrate followed by counterstaining with hematoxylin. Non-specific rabbit or mouse IgG substituting the primary antibodies served as the negative control.

Immunohistochemistry evaluation. Staining results were assessed in 10 fields ( $\times 200$ magnifications). To score a tumor cell as positive, cytoplasmic staining was required for $\mathrm{p}-\mathrm{mTOR}$ (Ser2448), p-4EBP1 (Thr70) and p-p70S6K (Thr389). The immunostaining was evaluated independently by a specialized pathologist who was blinded towards the patient's clinical features. For the analysis of the correlation between biomarkers, $\mathrm{H}$ scores for each staining were calculated by multiplying the intensity of the staining (0 to 3 ) and the fraction of positively stained tissue score (0 to 100 percent). For each biomarker, the cut-off point was determined by the median score of all cases, and a tumor was considered high expression when $\mathrm{H}$ scores were more than the median scores, as described previously [7].

Statistical analysis. Chi-square test and Fisher's exact test were used to test relationships between the expression of biomarkers and clinical/pathological factors. Kaplan-Meier curves and log-rank test were used to assess the differences in overall survival between positive and negative groups. Prognostic factors identified were further analysized in the multivariate analysis by a Cox proportional hazard model. Two-sided $p$ values of 0.05 or less were considered statistically significant. SPSS version 12.0 (SPSS Inc., Chicago, IL, USA) was used for statistic analysis.

\section{Results}

High levels of p-mTOR, p-4EBP1 and p-p70S6K expression were detected in $48.1 \%$ (37/77), 50.6\% (39/77) and 51.9\% (40/77) of all ICC tumors, respectively. Immunostaining of p-mTOR was cytoplasmatic and partly membranous, while p-p70S6K and p-4EBP1 was positive in the cytoplasm of cancer cells. And p-mTOR, p-4EBP1 and p-p70S6K showed no immunohistochemical staining in the surrounding stroma (Figure 1). In quantitative immunohistochemistry (by $\mathrm{H}$ score)

Table 1. Clinicopathological characteristics of the patients with ICC according to the activiation of mTOR pathway.

\begin{tabular}{|c|c|c|c|c|c|c|c|c|c|c|}
\hline & \multirow[b]{2}{*}{ Total } & \multicolumn{2}{|c|}{ p-mTOR expression } & \multirow[b]{2}{*}{$\mathbf{P}$} & \multicolumn{2}{|c|}{ p-4EBP1 expression } & \multirow[b]{2}{*}{$\mathbf{P}$} & \multicolumn{2}{|c|}{ p-p70S6K expression } & \multirow[b]{2}{*}{$\mathbf{P}$} \\
\hline & & $\begin{array}{l}\text { High } \\
\mathbf{n}=37\end{array}$ & $\begin{array}{c}\text { Low } \\
\mathrm{N}=40\end{array}$ & & $\begin{array}{l}\text { High } \\
n=39\end{array}$ & $\begin{array}{l}\text { Low } \\
n=38\end{array}$ & & $\begin{array}{l}\text { High } \\
n=40\end{array}$ & $\begin{array}{c}\text { low } \\
\mathbf{n}=37\end{array}$ & \\
\hline \multicolumn{11}{|l|}{ Age (y) } \\
\hline$<65$ & 60 & 28 & 32 & 0.648 & 27 & 33 & 0.063 & 31 & 29 & 0.926 \\
\hline$\geq 65$ & 17 & 9 & 8 & & 12 & 5 & & 9 & 8 & \\
\hline \multicolumn{11}{|l|}{ Gender } \\
\hline Female & 24 & 10 & 14 & 0.451 & 12 & 12 & 0.94 & 11 & 13 & 0.470 \\
\hline Male & 53 & 27 & 26 & & 27 & 26 & & 29 & 24 & \\
\hline \multicolumn{11}{|l|}{ Tumor stage } \\
\hline T1-T2 & 23 & 12 & 11 & 0.637 & 11 & 12 & 0.746 & 10 & 13 & 0.332 \\
\hline T3 & 54 & 25 & 29 & & 28 & 26 & & 30 & 24 & \\
\hline \multicolumn{11}{|c|}{ Lymph node status } \\
\hline Negative & 40 & 21 & 19 & 0.417 & 21 & 19 & 0.736 & 22 & 18 & 0.577 \\
\hline Positive & 37 & 16 & 21 & & 18 & 19 & & 18 & 19 & \\
\hline \multicolumn{11}{|c|}{ Tumor grade } \\
\hline G1-2 & 38 & 15 & 23 & 0.137 & 17 & 21 & 0.306 & 15 & 23 & 0.031 \\
\hline G3 & 39 & 22 & 17 & & 22 & 17 & & 25 & 14 & \\
\hline \multicolumn{11}{|l|}{ Recurrence } \\
\hline No & 47 & 20 & 27 & 0.227 & 23 & 14 & 0.466 & 26 & 21 & 0.459 \\
\hline Yes & 30 & 17 & 13 & & 16 & 14 & & 14 & 16 & \\
\hline
\end{tabular}




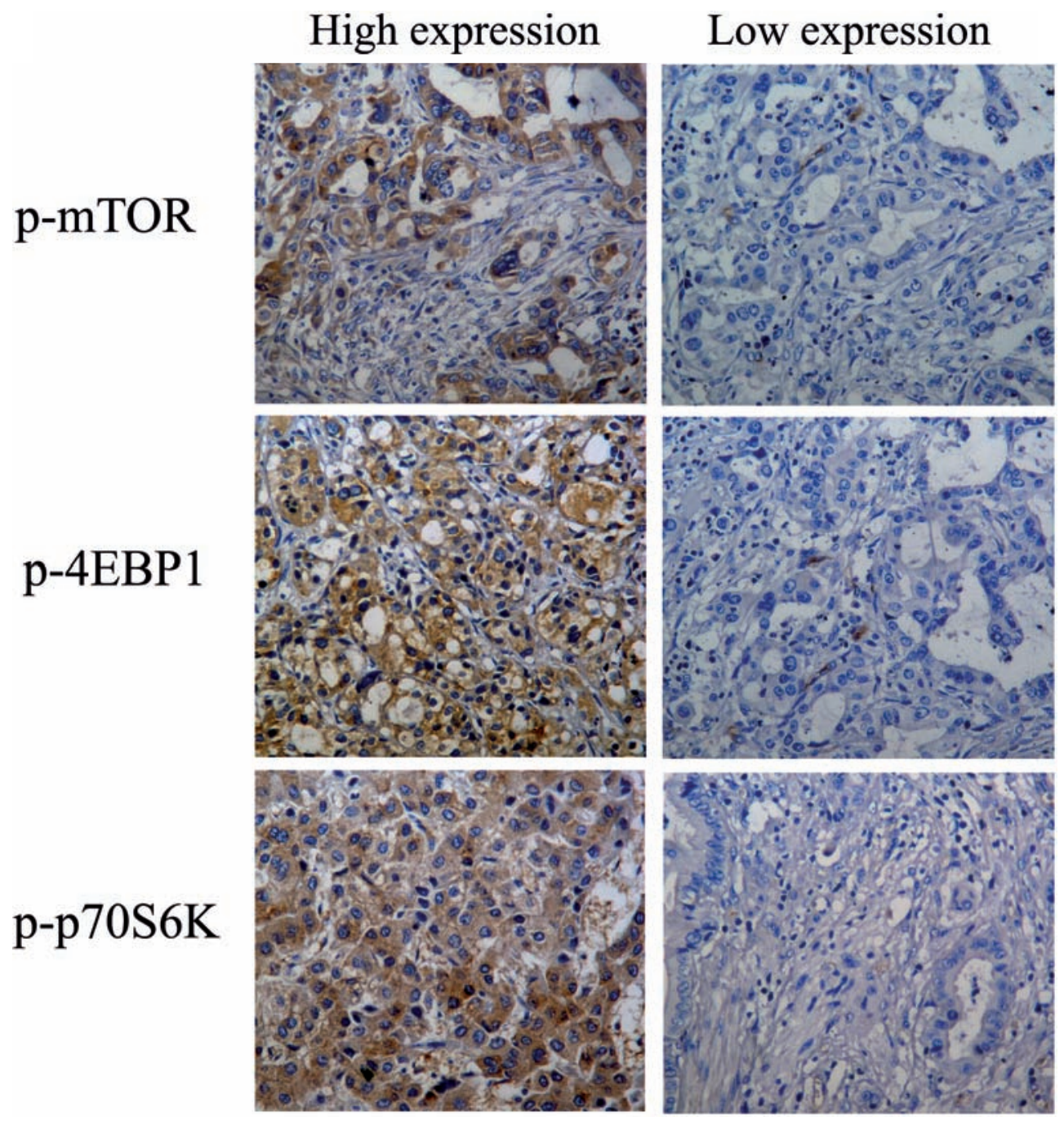

Figure1. Immunohistochemical staining of p-mTOR (Ser2448), p-4EBP1 (Thr70) and p-p70S6K (Thr389) in intrahepatic cholangiocarcinoma tissues.
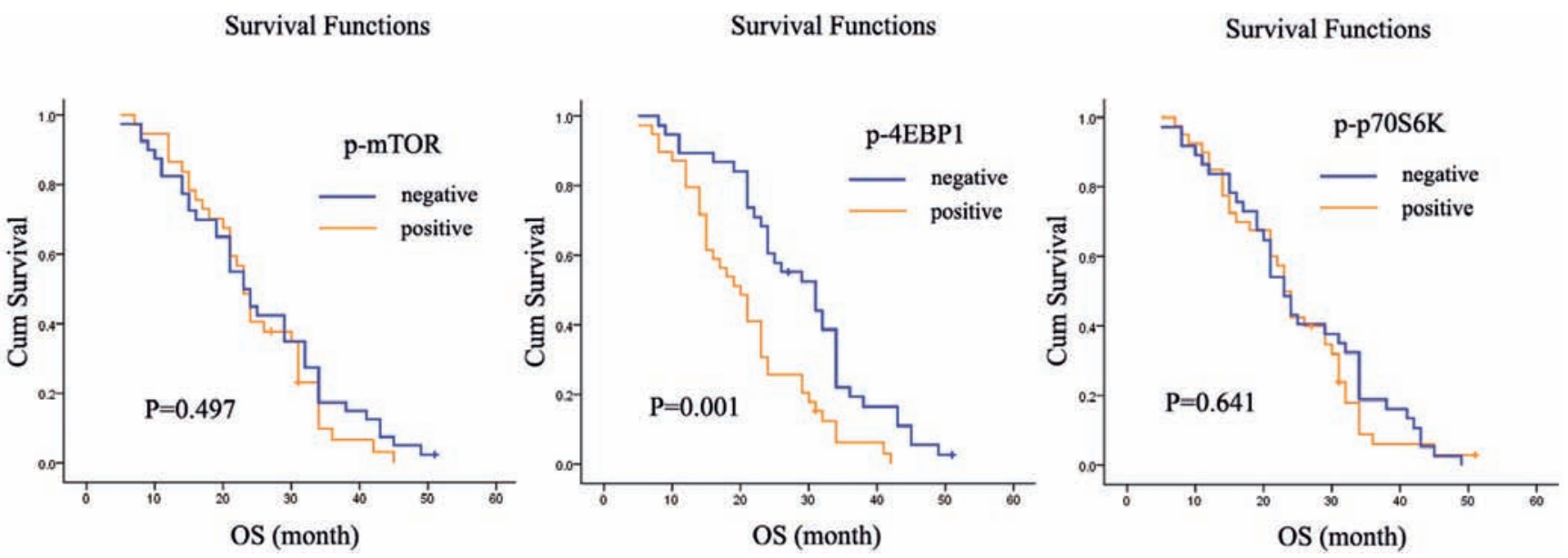

Figure 2. Kaplan-Meier survival analysis of intrahepatic cholangiocarcinoma according to p-mTOR (Ser2448), p-4EBP1 (Thr70) and p-p70S6K (Thr389) expression status. 
analysis, we found no association among the mTOR pathway proteins.

As seen in Table 1. No significant correlation was observed between $\mathrm{p}$-mTOR and p-4EBP1 overexpression with clinicopathological factors, including age, gender, tumor stage, lymph node status and grade. Tumors with high p-p70S6K expression had a trend to higher grade than those with low expression $(62.5 \%$ vs. $37.8 \%)$, a statistical significance was reached $(\mathrm{P}=0.031)$. However, high p-p70S6K expression was also not associated with the other clinicopathological factors.

Table 2 demonstrated the univariate analysis of the immunohistochemical results of the mTOR pathway proteins with regard to overall survival (OS). As seen in figure 2, a tendency towards shorter overall survival was observed in patients with high p-4EBP1 expression, but not with p-mTOR and p-p70S6K overexpression. In addition, multivariate analysis also showed that high p-4EBP1 expression was a significant independent prognostic factor for ICC patients (table 2).

\section{Discussion}

This study investigated three activated mTOR pathway proteins, $\mathrm{p}$-mTOR, $\mathrm{p}-4 \mathrm{EBP} 1$ and $\mathrm{p}$-p70S6K, in intrahepatic cholangiocarcinomas (ICC), in order to evaluate which subgroup of ICC patients might potentially benefit from mTOR pathway targeted inhibitors.

Our study found that high level of mTOR phosphorylated at Ser 2448 in $48.1 \%$ of the ICC tumors. It is believed that mTOR phosphorylated at Ser2448 is induced via the PI3 kinase/Akt signaling pathway and autophosphorylation [8]. Previous studies have demonstrated that mTOR phosphorylation at Ser2448 would play a key role in cellular growth and homeostasis, and is frequently altered in several tumors [9, 10]. Phosphorylation of mTOR in hepatocellular carcinoma (HCC) ranged from 15 to $41 \%$ [11]. Chung et al. [12] and Herberger et al. [13] found that p-mTOR was positive in $83.7 \%$ of the extrahepatic cholangiocarcinomas and $64 \%$ of biliary tract adenocarcinomas. Consistent with biliary tract adenocarcinomas, our data also indicated no association between mTOR phosphorylation with any of the clinical or pathologic variables. However, p-mTOR failed to predict overall survival of the patients, which is inconsistent with that in biliary tract adenocarcinomas.

We further detected the expression of two downstream effectors of activated mTOR pathway, p-4EBP1 and p-p70S6K, in ICC tumors. Similar with p-mTOR, about half of the ICC tumors indicated high p-4EBP1 and p-p70S6K expression, and no association was observed between $\mathrm{p}-4 \mathrm{EBP} 1$ and $\mathrm{p}-\mathrm{p} 70 \mathrm{~S} 6 \mathrm{~K}$ expression with any of the clinical or pathologic variables, except that high p-p70S6K expression correlated with poorly differentiated tumors.

Previous studies have found that phosphorylation of $\mathrm{p}$ 4EBP1 at the priming sites ( $\mathrm{Th} 37 / 46)$ was not an independent prognostic factor for many cancers such as ovarian cancers [14]. In this study, we used an antibody against p-4EBP1 at the subsequent phosphorylation site ( $\mathrm{Thr} 70$ ), which is potentially involved in the role of $\mathrm{p}-4 \mathrm{EBP} 1$ during carcinogenesis, including release of the eIF-4E and stimulation of cap-dependent translation of mRNA. Our overall survival analysis indicated that overexpression of p-4EBP1 was closely linked to poor prognosis of the ICC patients, and retained as an independent prognostic factor after adjustment for other clinical and pathologic factors in multivariate analysis. These findings suggest that phosphorylation at Thr 70 may be an evidence for activation of $\mathrm{p}-4 \mathrm{EBP} 1$ which impacts the prognosis of cancer patients.

Several studies have also identified that p-mTOR was not associated with expression of its downstream effectors, which is consistent with our findings. Recently, No et al. [15] found that p-4EBP1 expression was correlated with p-p70S6K expression in ovarian cancer. In this study, analysis of correlations among mTOR pathway proteins using immunostaining scores revealed no significant association between $\mathrm{p}-\mathrm{mTOR}$, $\mathrm{p}-4 \mathrm{EBP} 1$ and p-p70S6K. The discrepancy may be due to the different antibodies used and existing other regulatory mechanisms of mTOR proteins phosphorylation $\left.{ }^{[} 16,17\right]$.

In conclusion, our results showed the high prevalence of activation of mTOR pathway in ICC tumors, which suggests ICC patients might benefit from the personalized therapeutic

Table 2. Univariate and multivariate analysis for the prognosis

\begin{tabular}{|c|c|c|c|c|}
\hline \multirow{2}{*}{ Variable } & \multicolumn{2}{|c|}{ Univariate survival analyses } & \multicolumn{2}{|c|}{ Multivariate survival analysis } \\
\hline & HR for death $(95 \% \mathrm{CI})$ & $\mathbf{P}$ & Adjusted HR for death $(95 \% \mathrm{CI})$ & $\mathbf{P}$ \\
\hline Age & $1.258(0.710-2.215)$ & 0.427 & $1.100(0.588-2.055)$ & 0.766 \\
\hline Gender & $0.872(0.531-1.433)$ & 0.589 & $0.794(0.463-1.361)$ & 0.401 \\
\hline Tumor stage & $1.355(0.815-2.252)$ & 0.241 & $1.100(0.548-2.209)$ & 0.788 \\
\hline Lymph node status & $1.616(1.019-2.563)$ & 0.041 & $2.045(1.087-3.845)$ & 0.026 \\
\hline Tumor grade & $1.106(0.694-1.763)$ & 0.670 & $0.967(0.581-1.612)$ & 0.899 \\
\hline Recurrence & $1.096(0.676-1.777)$ & 0.709 & $1.400(0.788-2.488)$ & 0.252 \\
\hline High p-mTOR expression & $1.176(0.737-1.874)$ & 0.497 & $1.306(0.757-2.251)$ & 0.337 \\
\hline High p-4EBP1 expression & $2.285(1.406-3.715)$ & 0.001 & $3.105(1.751-5.505)$ & $<0.001$ \\
\hline High p-p70S6K expression & $1.126(0.709-1.789)$ & 0.641 & $0.842(0.490-1.446)$ & 0.532 \\
\hline
\end{tabular}


strategies with inhibitors of the mTOR pathway. In addition, $\mathrm{p}$ 4EBP1 phosphorylation at Thr 70 could be a useful biomarker for the prognostic evaluation of ICC patients.

\section{References}

[1] FARGES O, FUKS D. Clinical presentation and management of intrahepatic cholangiocarcinoma. Gastroenterol Clin Biol 2010; 34: 191-199. http://dx.doi. org/10.1016/j.gcb.2010.01.006

[2] SIRICA AE, DUMUR CI, CAMPBELL DJ, ALMENARA JA, OGUNWOBI OO, et al. Intrahepatic cholangiocarcinoma progression: prognostic factors and basic mechanisms. Clin Gastroenterol Hepatol 2009; 7: S68-78. http://dx.doi. org/10.1016/j.cgh.2009.08.023

[3] ZONCU R, EFEYAN A, SABATINI DM. mTOR: from growth signal integration to cancer, diabetes and ageing. Nat Rev Mol Cell Biol 2011; 12: 21-35. http://dx.doi.org/10.1038/ $\underline{\text { nrm3025 }}$

[4] YECIES JL, MANNING BD. mTOR links oncogenic signaling to tumor cell metabolism. J Mol Med (Berl) 2011; 89: 221228.

[5] WANDER SA, HENNESSY BT, SLINGERLAND JM. Nextgeneration mTOR inhibitors in clinical oncology: how pathway complexity informs therapeutic strategy. J Clin Invest 2011; 121: 1231-1241. http://dx.doi.org/10.1172/JCI44145

[6] ROYCHOWDHURY A, SHARMA R, KUMAR S. Recent advances in the discovery of small molecule mTOR inhibitors. Future Med Chem 2010; 2: 1577-1589. http://dx.doi. org $/ 10.4155 /$ fmc. 10.233

[7] SHINTO O, YASHIRO M, TOYOKAWA T, NISHII T, KAIZAKI R, et al. Phosphorylated smad2 in advanced stage gastric carcinoma. BMC Cancer 2010; 10: 652. http://dx.doi. org/10.1186/1471-2407-10-652

[8] NAVÉ BT, OUWENS M, WITHERS DJ, ALESSI DR, SHEPHERD PR. Mammalian target of rapamycin is a direct target for protein kinase B: identification of a convergence point for opposing effects of insulin and amino-acid deficiency on protein translation. Biochem J 1999; 344 Pt 2: 427-431.

[9] BROWN RE, ZHANG PL, LUN M, ZHU S, PELLITTERI PK, et al. Morphoproteomic and pharmacoproteomic rationale for mTOR effectors as therapeutic targets in head and neck squamous cell carcinoma. Ann Clin Lab Sci 2006; 36: 273-282.

[10] BROWN RE, ZOTALIS G, ZHANG PL, ZHAO B. Morphoproteomic confirmation of a constitutively activated mTOR pathway in high grade prostatic intraepithelial neoplasia and prostate cancer. Int J Clin Exp Pathol 2008; 1: 333-342.

[11] VILLANUEVA A, CHIANG DY, NEWELL P, PEIX J, THUNG $\mathrm{S}$, et al. Pivotal role of mTOR signaling in hepatocellular carcinoma. Gastroenterology 2008; 135: 1972-1983. http://dx.doi. org/10.1053/j.gastro.2008.08.008

[12] CHUNG JY, HONG SM, CHOI BY, CHO H, YU E, et al. The expression of phospho-AKT, phospho-mTOR, and PTEN in extrahepatic cholangiocarcinoma. Clin Cancer Res 2009; 15: 660-667. http://dx.doi.org/10.1158/1078-0432.CCR-08-1084

[13] HERBERGER B, PUHALLA H, LEHNERT M, WRBA F, NOVAK $S$, et al. Activated mammalian target of rapamycin is an adverse prognostic factor in patients with biliary tract adenocarcinoma. Clin Cancer Res 2007; 13: 4795-4799. http:// dx.doi.org/10.1158/1078-0432.CCR-07-0738

[14] NOSKE A, LINDENBERG JL, DARB-ESFAHANI S, WEICHERT W, BUCKENDAHL AC, et al. Activation of mTOR in a subgroup of ovarian carcinomas: correlation with p-eIF-4E and prognosis. Oncol Rep 2008; 20: 1409-1417.

[15] NO JH, JEON YT, PARK IA, KIM YB, KIM JW, et al. Activation of mTOR signaling pathway associated with adverse prognostic factors of epithelial ovarian cancer. Gynecol Oncol 2011; 121: 8-12. http://dx.doi.org/10.1016/j.ygyno.2010.12.364

[16] HAY N, SONENBERG N. Upstream and downstream of mTOR. Genes Dev 2004; 18: 1926-1945. http://dx.doi. org/10.1101/gad.1212704

[17] GINGRAS AC, RAUGHT B, GYGI SP, NIEDZWIECKA A, MIRON M, et al. Hierarchical phosphorylation of the translation inhibitor 4E-BP1. Genes Dev 2001; 15: 2852-2864. 